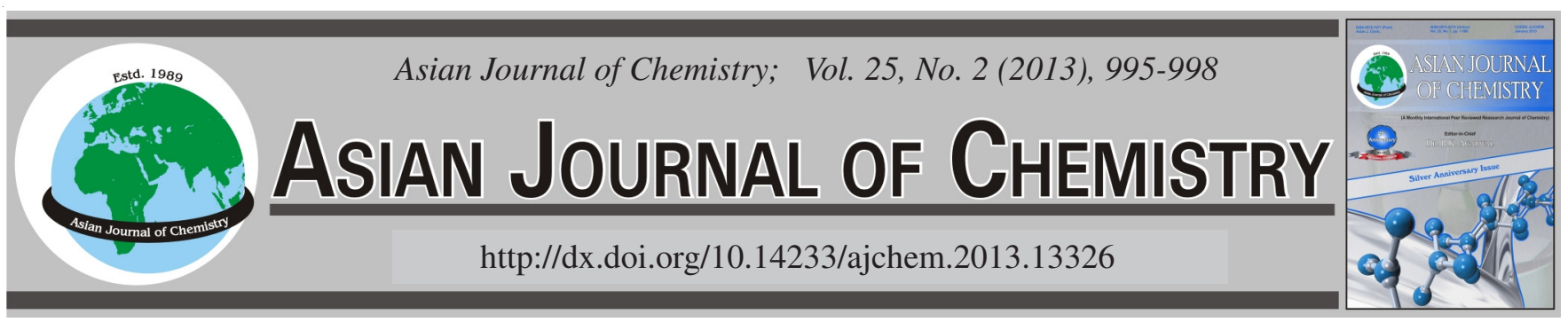

\title{
Phytochemical Analysis of Curcumin from Turmeric by RP-HPLC
}

\section{K.J. LEE ${ }^{1}$, S.D. CHOI ${ }^{2}$ and J.Y. MA ${ }^{1, *}$}

${ }^{1}$ Korean Institute of Oriental Medicine, TKM-Based Herbal Drug Research Group, 1672 Yuseongdae-ro, Yuseong-gu, Daejeon 305-811, South Korea

${ }^{2}$ Department of Chemical Engineering, Kangwon National University Samcheok Campus, Gangwon-do, Samcheok, 245-711, South Korea

*Corresponding author: Fax: +82 42 8689573; Tel: +82 42 8689466; E-mail: jyma@kiom.re.kr

Curcumin is isolated from turmeric. The effect of solvent composition on the total extraction yield of turmeric has been investigated using the dipping method. HPLC-UV diode-array (DAD), electrospray mass spectrometry (ESI) and TLC have been used simultaneously to analyze curcumin in a fresh turmeric extract. From the results, it is evident that the percentage of curcumin extracted from turmeric by pure methanol was higher than any aqueous methanol composition; although the total extraction yield was the highest in $100 \%$ water.

Key Words: Turmeric, HPLC, Extraction, Purification, Isolation.

\section{INTRODUCTION}

Turmeric, a member of the family Zingiberaceae, has long been extensively used to prevent and cure human diseases for thousands of years in many oriental countries because of its low toxicity and effective therapeutically performance. It belongs to the genus Curcuma that consists of hundreds of species of plants that possess rhizomes and underground root like stems. Turmeric, which belongs to a group of aromatic spices, had been originally used as a food additive in curries to improve the storage condition, palatability and preservation of food. Turmeric has also been used internally as a stomachic, tonic and blood purifier and externally in the preventation and treatment of skin diseases ${ }^{1}$. Most of the members of the genus Curcuma are distributed throughout tropical and subtropical regions of the world and are widely cultivated in Asian countries $^{1,2}$. Also, the quantity and quality of safety and efficacy data on the herbal medicines are far from sufficiency to satisfy the criteria needed to support its world-wide use. The rhizomes are light yellow and possess a camphoraceous odor and it has been found to be a rich source of diarylheptanoids, namely, curcuminoids $^{2-4}$. All three impart [curcumin (1), demethoxycurcumin (2) and bisdemethoxycurcumin (3)] the hallmark yellow pigmentation to the turmeric plant and particularly to its rhizomes ${ }^{3,4}$. These curcuminoids are active constituents of turmeric and have been shown to contribute to many of the medicinal properties, like anticancer, antioxidant, anti HIV, antiviral and antiinflammatory activities ${ }^{5-10}$. The quantitative analysis of curcuminoids, specially curcumin in turmeric is very important to determine the quality of plant material or its processed products. A large number of reports and several reviews have appeared on the analysis, identification and separation of individual curcuminoids ${ }^{11-14}$. However, all of these investigations did not cover the effect of solvent-composition on total extraction yield of turmeric. In the present work, the effect of solvent-composition on total extraction yield has been studied. The quantitative analysis of curcumin in different extraction has also been studied using RP-HPLC with reference to standard curcumin.

\section{EXPERIMENTAL}

Asian turmeric (country of origin: China) was acquired from the Korean Institute of Oriental Medicine, Center for Herbal Medicine Improvement Research Principal Research Scientist Center, Korea in May, 2011. Standard samples were prepared by dissolving $2 \mathrm{mg}$ of the standard chemical (curcumin: sigma-aldrich, china) in $4 \mathrm{~mL}$ of methanol and adjusting the concentration to $500 \mathrm{ppm}$. The HPLC-grade methanol and analytical grade hexane, acetonitrile, dichloromethane, ethyl acetate, acetic acid were purchased from J.T. Baker (USA). The twice distilled water was filtered by a pump (Division of Millipore, Waters, Milford, MA, USA).

Solvent extraction: Using the turmeric standardize, dried samples without particles (sieving $<30 \mu \mathrm{m}$ ) was prepared and stored at low temperature and moisture content $21.60 \%$ were used. Then $3 \mathrm{~g}$ (for each extraction) of powdered turmeric was used for extraction using solvent-composition $(200 \mathrm{~mL}$ for each extraction) of increasing polarity, i.e., $100 \%$ methanol, 
$80 \%$ aqueous methanol, $60 \%$ aqueous methanol, $40 \%$ aqueous methanol, $20 \%$ aqueous methanol and finally $100 \%$ water by dipping method for $4 \mathrm{~h}$ at $25^{\circ} \mathrm{C}$. Each extract was filtered, concentrated under vacuum using rotavapor and refrigerated for $48 \mathrm{~h}$. Then the samples were frozen dry and the total extraction yield was calculated. Each sample was filtered through a $0.2 \mu \mathrm{m}$ membrane filter prior to HPLC analysis.

HPLC, LC-MS and TLC analysis: The experiments were performed with a Dionex HPLC system equipped with an ultimate 3000 pump, ultimate diode array detector (DAD), $10 \mu \mathrm{L}$ sample injector loop (Dionex ID $\times$ L $0.18 \mathrm{~mm} \times 550$ mm USA) and Chromeleon data acquisition system (Dionex version 7.0.1.272). The standard curcumin was confirmed by LC-MS analysis (Varian: 500-MS IT Mass Spectrometer). The chromatographic columns used in this experiment are commercially available, obtained from Shimadzu RP-column (150 mm $\times 2.1 \mathrm{~mm} 3.5 \mu \mathrm{m}$ Pore Dia $100 \AA \mathrm{C}_{18}$ Japan). The injection volume was $10 \mu \mathrm{L}$ and the flow rate of the mobile phase was $1.0 \mathrm{~mL} / \mathrm{min}$. The wavelength of the UV detector was fixed at 425 and $254 \mathrm{~nm}$. The mobile phase solvents were A (water/ acetic acid $=99.9 / 0.1 \mathrm{v} / \mathrm{v}$ ) and solvent B (acetonitrile/acetic acid $=99.9 / 0.1 \mathrm{v} / \mathrm{v}$ ). The run time was $20 \mathrm{~min}$ and isocratic method was applied (solvent A $40 \%$ and B $60 \%$ ). The analyses were performed by a silica XHL TLC plates glass $(5 \mathrm{~mm} \times 10$ $\mathrm{cm} 250 \mu \mathrm{m}$ Sorbent Tech. USA) developed with a mobile phase composed of chloroform-methanol $(97: 3 \mathrm{v} / \mathrm{v})$ and stained with a $10 \% \mathrm{H}_{2} \mathrm{SO}_{4}$ reagent to detect the turmeric extract.

Extraction yield: Depending on the extraction method, each extract underwent decompression concentration to rotary evaporate the solvent in a refrigerated vapor trap for $48 \mathrm{~h}$. Each experiment was performed in two replicates and the data were subjected to calculations of means \pm SE. Then the extract samples were expressed as a percentage of the weight and the extraction yield was measured using the following equation:

$$
\text { Exraction yield }(\%, w / w)=\frac{\text { Exracts dry weight }}{\text { Sample dry weight }} \times 100
$$

\section{RESULTS AND DISCUSSION}

In this work, isolated and analysis of curcumin from turmeric using reverse phase high performance liquid chromatography (RP-HPLC). For extraction of the curcumin, different aqueous methanol were used. When the concentrations of methanol in aqueous solvent were increasing, the total yield decreases (Table-1), because the pure water could be extracted the more polar materials than the aqueous methanol. The maximum measured extraction yield was $22.70 \%$. The extracted samples were analyzed by RP-HPLC, on-line HPLCUV-MS and TLC. The commercially available standard compound, curcumin (1), showed three peaks. The peak area of curcumin was $80 \%$ of the total area; other two peaks were identified as compounds $\mathbf{2}$ and $\mathbf{3}$. Commercially available curcumin mixture contain 79.84-80\% curcumin, 15-16\% demethoxycurcumin, 2.96-3\% bisdemethoxycurcumin and etc. $2.2 \%)^{15}$.

Compound curcumin (1) was confirmed by both chromatographic and MS data identification of curcuminoid analogues in all items using HPLC analysis was based on retention times

\begin{tabular}{cccc}
\hline \multicolumn{4}{c}{ TABLE-1 } \\
QUANTITATIVE ANALYSIS OF CURCUMIN IN \\
DIFFERENT EXTRACT FROM TURMERIC \\
\hline $\begin{array}{c}\text { Oriental } \\
\text { medicine } \\
\text { name }\end{array}$ & $\begin{array}{c}\text { Extraction } \\
\text { solvent (\%) }\end{array}$ & $\begin{array}{c}\mathrm{t}_{\mathrm{R}}(\mathrm{min}) \text { of } \\
\text { curcumin at } \\
425 \mathrm{~nm}\end{array}$ & $\begin{array}{c}\text { Total } \\
\text { extraction } \\
\text { yield (\%) }\end{array}$ \\
\hline \multirow{5}{*}{ Turmeric } & Water (100) & 1.173 & 22.7 \\
(Curcuma & Methanol (20) & 1.170 & 16.8 \\
longa) & Methanol (40) & 1.198 & 18.5 \\
& Methanol (60) & 1.164 & 14.8 \\
& Methanol (80) & 1.159 & 13.7 \\
\hline
\end{tabular}

and on the comparison of their UV-detector and wavelengths of maximum absorption with those of representative standards as previously described method. Quantification was performed on the basis of linear calibration plots of the UV absorption peak area at 420-425 nm (Fig. 1) $)^{2}$. To determine the optimum flow rate in this HPLC system, different flow rates were used. When the flow rate increases, the retention time decreases. The experimental parameters in the equilibrium curves were estimated by linear (eqn. 2) and polynomial (eqn. 3) regression analyses.

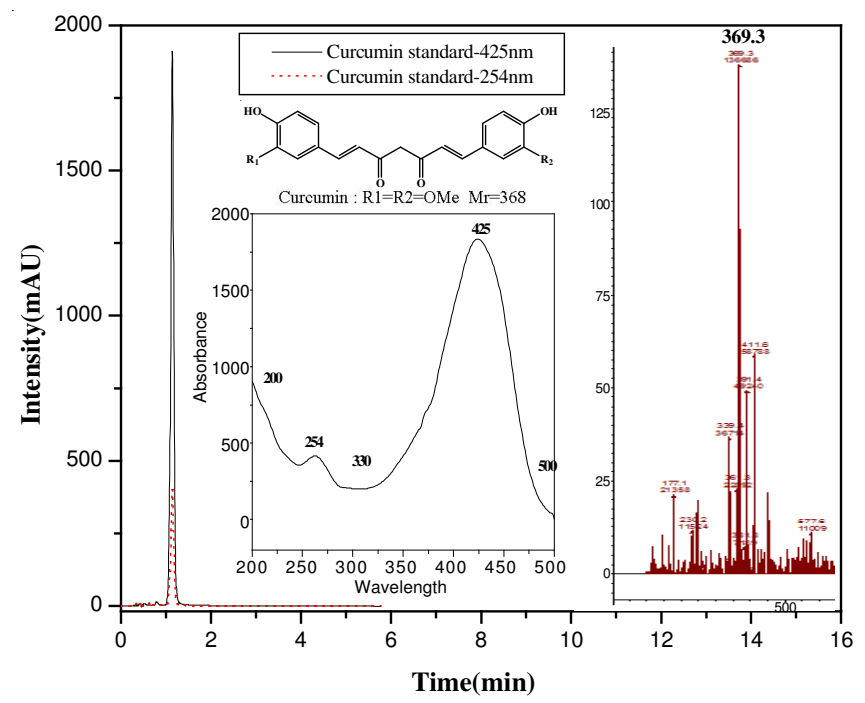

Fig. 1. Analysis of UV detector and LC-MS from curcumin standard

$$
\begin{gathered}
y=a x+b \\
y=a x^{2}+b x+c \\
r^{2}=1-\frac{\sum_{i=1}^{n}\left(y_{i}-y\left(x_{i}\right)\right)^{2}}{\sum_{i=1}^{n}\left(y_{i}-<y_{i}>\right)^{2}}
\end{gathered}
$$

where $\mathrm{a}, \mathrm{b}, \mathrm{c}$ are the parameters, $\mathrm{r}^{2}$ is the regression coefficient (eqn. 4). The experimented and calculated value with different flow rates were shown in Fig. 2. From the result, the calculated data is well fitted with the experimented data. The regression coefficient (0.9821) was above by polynomial in this regression analyses. Fig. 3(a-b) show the chromatograms with different extraction solvents, respectively (analysis water 100, 40 and 


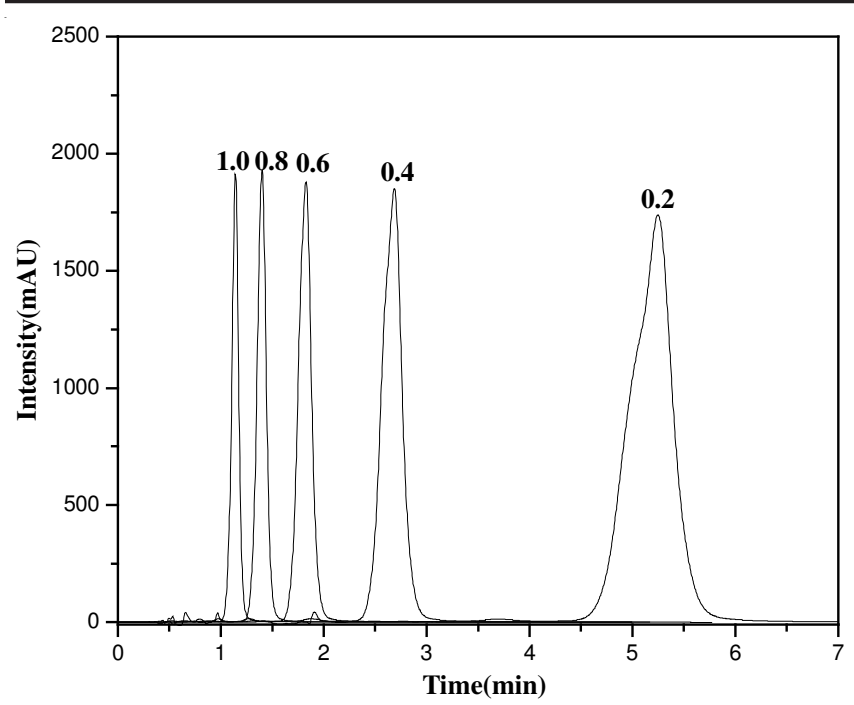

(a)

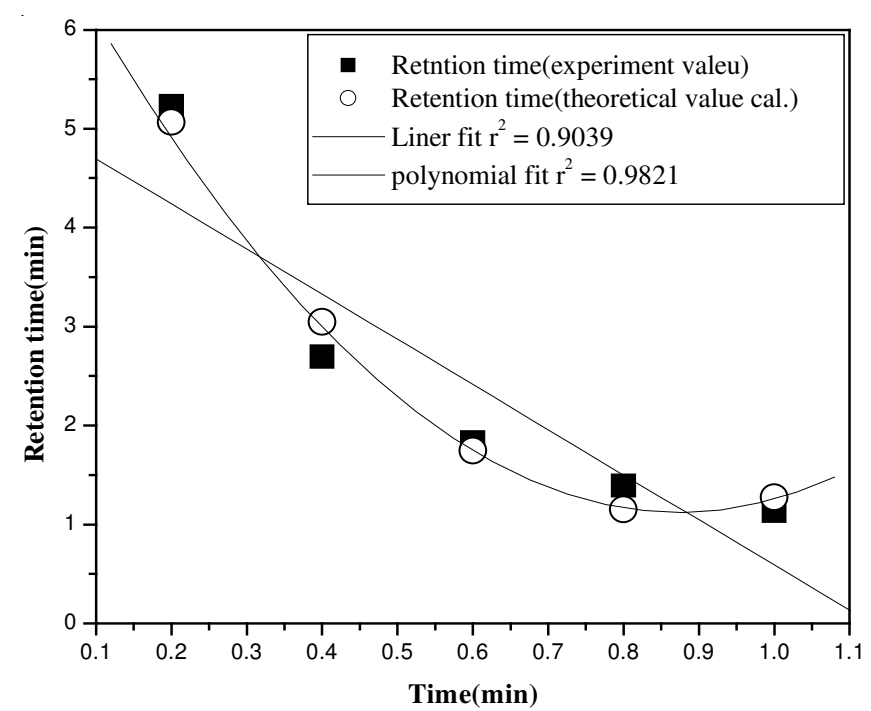

(b)

Fig. 2. Shift of the retention time of curcumin with mobile phase flow rates using RP-HPLC. (a : Chromatogram, b : Numerical analysis)

$20 \%$ aqueous methanol solvent extract are not shown here). The extraction efficiency and chemical properties of turmeric using dipping method for $4 \mathrm{~h}$ at $25^{\circ} \mathrm{C}$. The dried turmeric was pulverized and extracted by each pure water $100 \%$, methanol $100 \%$ and aqueous methanol of composition (60 and $80 \%$ ) using $200 \mathrm{~mL}$ of solvent for each extraction. From Fig. 3, it is evident that curcumin content is the maximum in $100 \%$ methanol extract of turmeric. A TLC has been done to isolate curcumin from methanol extract. In the extraction solvents, the methanol is the better effective solvent for extraction the curcumin from turmeric. It is evident that the percentage of curcumin extracted from turmeric by pure methanol was higher than any aqueous methanol composition, although the total extraction yield was the highest in $100 \%$ water. In general, selection of extract solvent for extraction is very important. Polar solvent make soluble polar compound and thus extraction yield will be higher in that particular solvent. For turmeric, water is the best fitted solvent to get the highest extraction yield. The isolated peak [fraction 1 in Fig. 3 (a)] was identified

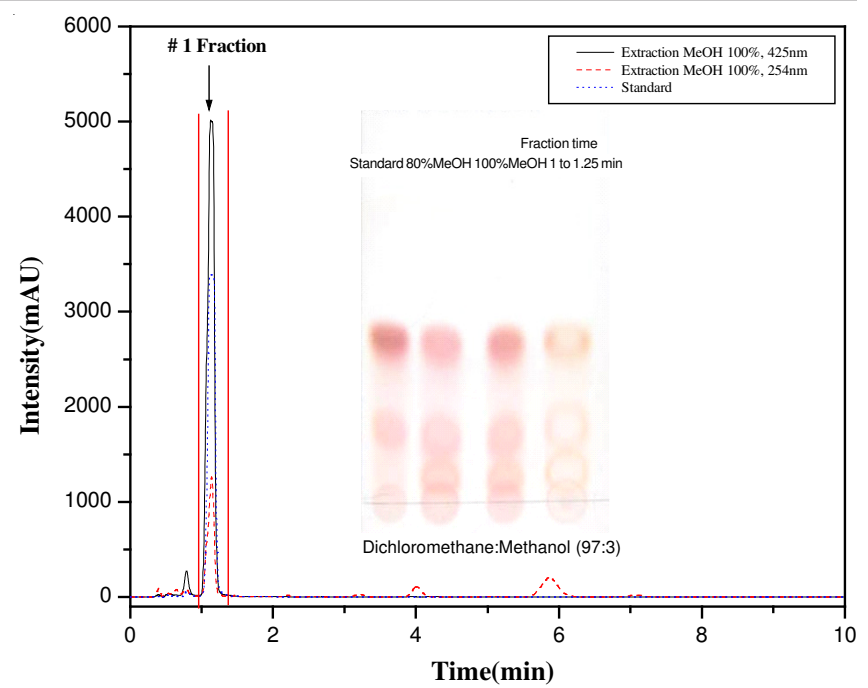

(a)

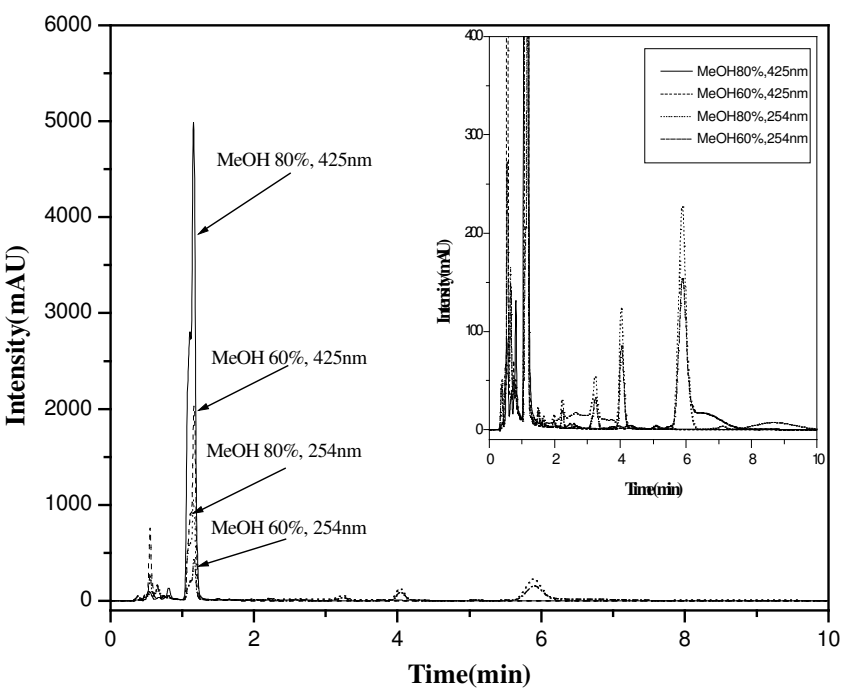

(b)

Fig. 3. Comparison of chromatogram by pure methanol and aqueous methanol composition. (a: methanol $100 \%$ and TLC analysis, b: aqueous methanol 80 and $60 \%$ analysis)

as curcumin from the HPLC profile as compared with the same of the standard. Among them, curcumin has been unambiguously identified, based on their UV spectra, mass spectra and retention times in comparison with the data of standard compounds. On-line HPLC-UV diode-array and electrospray mass spectrometry (MS) (Fig. 4) have been used simultaneously to analyze curcuminoids such as major curcumin fraction \# 1 in a fresh turmeric extract. MS spectra of the isolated peak also confirmed the presence of curcumin.

\section{Conclusion}

The curcuminoids, such as major curcumin, in a fresh turmeric were extracted and simultaneously identified using HPLC-UV-MS and TLC. From the result, the flow rate was increased, the retention time decreased. The calculated data is well fitted with the experimental data. The regression coefficient $(0.9821)$ was above nonlinear (polynomial) in this regression analyses. The results showed the effect of solventcomposition on total extraction yield from turmeric. Based on our investigations, anyone can choose the best solvent for 


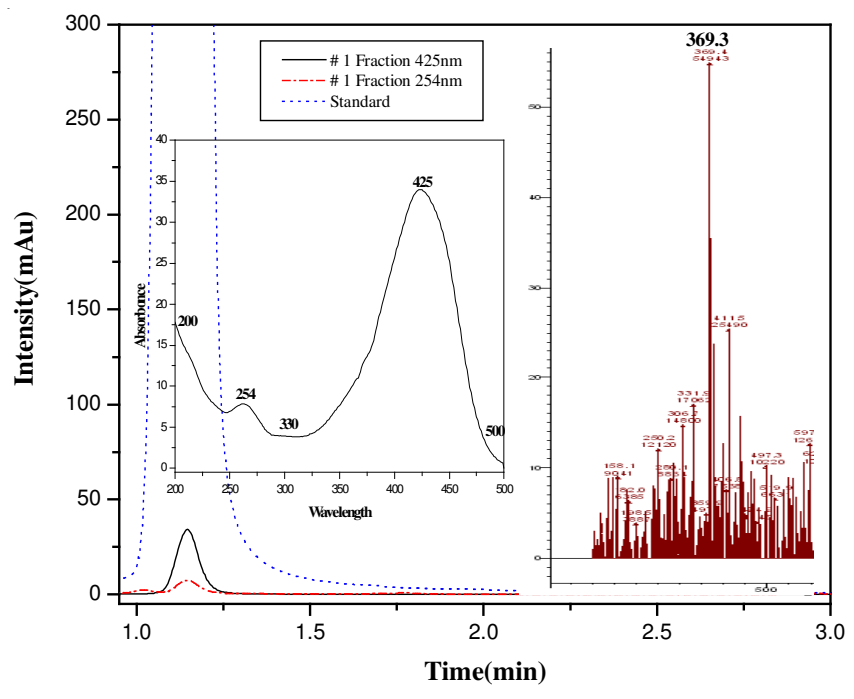

Fig. 4. HPLC profile and MS spectra of the isolated peak from methanol $100 \%$ extraction of turmeric. (Shown the Fig. 3 fraction \# 1)

extraction from turmeric at first time, no need to waste time for trial and error method. It is also found that curcumin content is the highest in $100 \%$ methanol extract, although total extraction yield is the maximum in $100 \%$ water extract. These results will form a database for investigating the constituents of natural products and the resources of pharmaceutical, nutrition and cosmetic products.

\section{ACKNOWLEDGEMENTS}

This study achieved in KIOM, TKM-Based Herbal Drug Research Group, Also, this work was supported by the "Study on drug efficacy enhancement using bioconversion for herbal medicines" (K12050) project, the authors gratefully acknowledged.

\section{REFERENCES}

1. J.C. Tilak, M. Banerjee, H. Mohan and T.P. Devasagayam, Phytother. Res., 18, 798 (2004).

2. X.G. He, L.Z. Lin, L.Z. Lian and M. Lindermaier, J. Chromatogr. A, 818, 127 (1998).

3. X. Sun, C, Gao, W. Cao, X. Yang and E. Wang, J. Chromatogr., 962, 117 (2002).

4. B.B. Aggarwal, A. Kumar and A.C. Bharti, Anticancer Res., 23, 363 (2003).

5. S. Gilda, M. Kanitkar, R. Bhonde and A. Paradkar, Food Sci. Technol., 43, 59 (2010).

6. S. Pandit, H.J. Kim, J.E. Kim and J.G. Jeon, Food Chem., 126, 1565 (2011).

7. R.K. Singh, D. Rai, D. Yadav, A. Bhargava, J. Balzarini and E.D. Clercq, Eur. J. Med. Chem., 45, 1078 (2010).

8. W. Thongchai, B. Liawruangrath and S. Liawruangrath, Food Chem., 112, 494 (2009).

9. B.B. Aggarwal and K.B. Harikumar, Int. J. Biochem. Cell Biol., 41, 40 (2009).

10. G.K. Jayaprakasha, L.J.M. Rao and K.K. Sakariah, Food Sci. Technol., 16, 533 (2005).

11. H.H. Tomesen and J. Karlsen, J. Chromatogr., 259, 367 (1983).

12. H.D. Jang, K.S. Chang, Y.S. Huang, C.L. Hsu, S.H. Lee and M.S. Su, Food Chem., 103, 749 (2007).

13. P.Y. Zhan, X.H. Zeng, H.M. Zhang and H.H. Li, Food Chem., 129, 700 (2011).

14. J.H. Lee and M.G. Choung, Food Chem., 124, 1217 (2011).

15. P. Anand, S.G. Thomas, A.B. Kunnumakkara, C. Sundaram, K.B. Harikumar, B. Sung, S.T. Tharakan, K. Misra, I.K. Priyadarsini, K.N. Rajasekharan and B.B. Aggarwal, Biochem. Pharm., 76, 1590 (2008). 